\title{
Tipo de agresión resultado de un problema y conflicto en la población adolescente de siete instituciones públicas de educación media de San Juan de Pasto
}

\section{Recibido: \\ 23 de Febrero}

de 2015

Aprobado:

19 de Mayo de 2015

* Enfermera Magister. Docente Investigadora.

Coordinadora de Autoevaluación e Internacionalización. Universidad Mariana. Pasto, Colombia. Correo electrónico: cchaves27@gmail.com

** Enfermera Magister. Docente Investigadora. Coordinadora de Salud Pública.

Universidad Mariana. Pasto,

Colombia. Correo electrónico: magalymorales2009@gmail. com

**** Enfermera Magister. Docente Investigadora. Coordinadora de Proyección Social. Universidad Mariana. Pasto, Colombia. Correo electrónico: nortdena@yahoo. es

**** Descriptores en Ciencias de la Salud (DeCS), en la página http:// decs.bvs.br/E/ homepagee.htm de la Biblioteca virtual en salud del proyecto BIREME, de la Organización Mundial de la Salud y de la Organización Panamericana de Salud.

\author{
Claudia Amanda Chaves* \\ Rosa Magaly Morales Chincha** \\ Nohora del Carmen Ortega Cadena**
}

\section{RESUMEN}

Objetivo: determinar el tipo de agresión que resulta de un problema o conflicto en adolescentes de siete instituciones públicas de educación media de San Juan de Pasto, Nariño, periodo 2013 - 2014. Materiales y Métodos: estudio cuantitativo con diseño descriptivo. La información se obtuvo a través de un cuestionario diseñado y modificado por las investigadoras y autoadministrado por los estudiantes de siete instituciones públicas de educación media jornada de la mañana. La población estuvo conformada por 5537 adolescentes entre las edades de 10 a 17 años, se realizó un muestreo probabilístico aleatorio simple y a través de la afijación proporcional se tomó una muestra de 774 estudiantes. Resultados: se encontró que los lugares con mayor prevalencia donde se presenta el tipo de agresión entre estudiantes son: cerca al colegio, al salir de clase, al interior del aula cuando no está el profesor y por último en pasillos y baños. El tipo de agresión más frecuente es la verbal, que por lo general se origina cuando se habla mal de la persona o por decir palabras ofensivas; las patadas, golpes y peleas entre grupos como tipo de agresión física no reportaron una frecuencia alta entre los adolescentes. Conclusión: el matoneo continúa presente en las instituciones de educación media convirtiéndose en una problemática social que ha sido tratada por diferentes sectores con el fin de encontrar una solución, pero los esfuerzos no están dando resultados; por ello, se debe continuar fortaleciendo el diseño de estrategias e implementación de modelos de convivencia sana orientadas a padres, educadores y estudiantes para la solución de conflictos y comprensión de consecuencias.

PALABRAS CLAVE: adolescente, estudiantes, problemas sociales, violencia ${ }^{* * * *}$.

Para citar este artículo / To reference this article / Para citar este artigo

Chaves CA, Morales-Chincha RM, Ortega-Cadena NdelC. Tipo de agresión resultado de un problema y conflicto en la población adolescente de siete instituciones públicas de educación media de San Juan de Pasto. Rev. cienc. cuidad. 2015; 12(1): 76-92. 


\section{Type of aggression result of a problem and conflict in the adolescent population of seven public institutions of secondary education in Pasto city}

\section{ABSTRACT}

Objective: to determine the type of aggression that results from a problem or conflict among adolescents in seven public institutions of education Media Pasto, Nariño. Period 2013 - 2014. Materials and Methods: descriptive quantitative study design, the information was obtained through a questionnaire designed and modified by the researchers and selfadministered by students from seven public institutions of secondary education day in the morning. The population was composed of 5537 adolescents between 10-17 years old. It was performed a simple random probability sampling. Through the proportional allocation it showed a sample of 774 students. Results: we found that areas with higher prevalence which presents the type of aggression among students are: close to school, after school, as well as in the class when the teacher isn't in class and finally, it happens in hallways and restrooms. The most common types of aggression are verbal and they start when someone says bad things about the other person, and by saying offensive words; kicks, punches and fights between groups and types of physical assault did not report a high frequency among adolescents. Conclusion: bullying continues in secondary education institutions becoming a social problem where different sectors have tried to solve it but the efforts are not working; for this reason, it is necessary to continue to strengthen in parents, educators and students strategies and models of healthy conflict solution to understand the possible consequences.

KEYWORDS: adolescent, students, social problems, violence. 


\section{Tipo de agressão resultado de um problema e conflito na população adolescente de sete instituições públicas de Pasto ensino secundário}

\section{RESUMO}

Objetivo: para determinar o tipo de agressão que resulta de um problema ou conflito entre os adolescentes em sete instituições públicas de ensino de mídia Pasto, Nariño, período de 2013 - 2014. Materiais e Métodos: estudo quantitativo descritivo, a informação foi obtida através de um questionário e modificado pelos pesquisadores e auto-administrada por estudantes de sete instituições públicas de ensino dia normal na parte da manhã. A população consistiu de 5.537 adolescentes com idades entre 10-17 anos foi realizada uma amostragem probabilística aleatória simples e através da alocação proporcional eu mostrar uma amostra de 774 alunos. Resultados: verificou-se que as áreas com maior prevalência que apresenta o tipo de agressão entre os estudantes estão perto de escola, depois da escola, bem como na classe quando não é o professor e, finalmente, em corredores e banheiros, tipo mais comuns são a agressão verbal e originam quando falar mal da pessoa, como a dizer palavras ofensivas; chutes, socos e brigas entre grupos e tipos de agressão física não relatou uma alta freqüência entre os adolescentes. Conclusão: o assédio moral continua em instituições de ensino secundário tornando-se um problema social onde os diferentes setores têm tentado resolvê-lo, mas os esforços não estão funcionando; porque deveria continuar a reforçar a pais, educadores e estudantes; estratégias e modelos de solução de problemas de vida saudável e compreensão das consequências.

PALAVRAS-CHAVE: adolescentes, estudantes, problemas sociais, violência. 


\section{INTRODUCCIÓN}

$\mathrm{L}$ a adolescencia en una etapa de la vida en la cual se manifiestan experiencias emocionales de toda índole, quizá por la serie de cambios físicos, sicológicos y emocionales que conllevan a percibir un sinnúmero de experiencias y sentimientos, entre ellos los de violencia, traducidos a los problemas y conflictos que son aspectos difíciles de manejar y que muchas veces obstaculizan la consecución de un fin, debido a que estas situaciones perturban la paz y la armonía de quienes los viven, desestabilizando su equilibrio personal y grupal (1).

"La adolescencia es esencialmente una época de cambios, donde la acompañan enormes variaciones físicas y emocionales. Es realmente un período vulnerable para la aparición de conductas de riesgo, las cuales pueden encontrarse por sí solas o concurrir $y$ traer consecuencias para la salud, económicas y sociales; es importante comprender el entorno en que viven y cómo afecta dicho entorno el desarrollo del adolescente, la conducta y las relaciones sociales. Estos entornos, entre ellos la familia, los grupos de pares, la escuela, el trabajo y las actividades de ocio, están al mismo tiempo en un estado de cambio constante" (1).

Según la Organización Mundial de la Salud (OMS), la adolescencia es el periodo comprendido entre los 10 y los 19 años; es una etapa compleja de la vida que marca la transición de la infancia al estado adulto y con ella se producen cambios físicos, psicológicos, biológicos, intelectuales y sociales (1).

Gómez et al. (2) asumen la violencia en escolares como una situación social en la que uno o varios escolares toman como objeto de su actuación injustamente agresiva a otro/a compañero/a y lo someten, por tiempo prolongado, a agresiones físicas, burlas, hostigamiento, amenaza, aislamiento social o exclusión social.

Es importante señalar que la violencia y/o el maltrato ente iguales genera cierto tipo de agresiones, las cuales se clasifican en verbales y físicas y se manifiestan en escenarios específicos que el adolescente escoge para cometer daño a un tercero.

El estudio de Ramos (3) menciona que el 5,7 \% de los estudiantes españoles reconoce agredir cada semana a alguno de sus compañeros con insultos, golpes, acoso psicológico o aislamiento del grupo. Casi un $6 \%$ ha vivido directamente el fenómeno conocido como bullying, el $90 \%$ es testigo de una conducta de este tipo en su entorno y el 30\% ha participado en alguna ocasión ya sea como víctima o como agresor. Entre el $25 \%$ y el $30 \%$ de los estudiantes de primer ciclo afirma haber sido víctima alguna vez de agresiones. Sólo 1 de cada 3 individuos que lo sufren son capaces de denunciarlo.

Este tipo de comportamientos que adquieren los adolescentes preocupa a padres, profesores y comunidad en general porque la constancia de estas conductas provoca una grave perturbación, tanto para las familias como para las instituciones y la sociedad. Es en este periodo de vida donde los jóvenes se ven enfrentados a un sinnúmero de problemas sociales, generando desacuerdos, discordancias y agresividad, lo cual deja también muchas consecuencias, a tal grado de que no sólo afecta a los involucrados sino también, de manera indirecta, a quienes los rodean.

Es esencial mencionar que la violencia escolar no solamente es cuestión del género masculino, como muchos piensan, puesto que las mujeres también han retomado una conducta agresiva y desenfrenada que se evidencia en agresiones verbales y también físicas.

El trabajo de Paredes et al. (4), revela que la principal razón de las mujeres colombianas para agredir a otras u otros es para desquitarse, sólo porque quiso o sólo por molestar; perciben la indefensión y debilidad en sus víctimas y ello les lleva a provocar dolor en forma intencional, lo que las hace sentir satisfechas, poderosas y cómodas con su comportamiento.

A nivel mundial se han unido esfuerzos para combatir este flagelo, que cada vez se acrecienta más. Es así como en Finlandia el estudio de Salmivall (5) evaluó el programa denominado KiVa (programa antiintimidación basado en la investigación), el cual ha trabajado en un ensayo controlado aleatorio con 117 escuelas de intervención y 117 escuelas de control. El programa ha demostrado que reduce significativamente tanto el acoso como la victimización, propia y reportada por pares, además de influir en forma múltiple en la victimización, incluida la verbal, física y el ciber-acoso, KiVa ganó el Premio Europeo de Prevención del Delito en 2009 (5). 
Tipo de agresión resultado de un problema y conflicto en la población adolescente de siete instituciones públicas de educación media de San Juan de Pasto • Claudia Amanda Chaves, Rosa Magaly Morales-Chincha, Nohora del Carmen Ortega-Cadena

ISSN 1794-9831

E-ISSN 2322-7028

Vol. 12 No. 1

Ene - Jun 2015

Cúcuta, Colombia
En Colombia el estudio de De Zubiria et al. (6) cubrió 807 centros de enseñanza, donde fueron entrevistados más de 82.000 estudiantes y estableció que el $56 \%$ de los encuestados ha sido robado al menos una vez y el $32 \%$ ha sido objeto de golpes o de algún maltrato físico por parte de otros estudiantes, dentro de las instalaciones educativas; 4.330 dijeron haber requerido atención médica después de la agresión y 2.580 aseguraron que quien los amenazó portaba un arma.

En Pasto, el trabajo de Redondo y Guevara (7) revela la prevalencia de la conducta prosocial que fue del $34.9 \%$. Además, las mujeres puntuaron significativamente más alto que los hombres en conducta prosocial y más bajo en conducta agresiva, con una proporción de mujeres prosociales significativamente mayor que la de los hombres.

El Gobierno Nacional de Colombia, preocupado por los sucesos de matoneo que cada vez aumentan más en el país, sancionó en el mes de marzo la Ley 1620 de 2013 (8), la cual pretende, entre otras cosas, atender preventivamente situaciones de violencia escolar como el bullying.

Con esta ley y el apoyo de todos los actores involucrados, se tiene la convicción de que el conflicto se puede resolver, teniendo en cuenta que la unión de las personas, las instituciones, los grupos sociales y las familias conduzca a un acto de intervención en la adolescencia, para producir, mediante una actitud positiva, una reorganización de la sociedad y así vislumbrar un reconocimiento del proyecto de vida por parte de los adolescentes.

Por todo lo anterior, llevar a cabo este proyecto desde la enfermería constituyó una importante incursión en ámbitos antes no explorados, pero que, en definitiva, son de su competencia, pues permiten a la disciplina retomar sus propios referentes teóricos e integrar el cuidado hacia los adolescentes como personas con sus propias identidades, valores y comportamientos $\mathrm{y}$, de esta forma, custodiar y contribuir al desarrollo humano de los mismos, con intervenciones objetivas desde su propia realidad.

\section{MATERIALES Y MÉTODOS}

Esta es una investigación cuantitativa de tipo descriptivo. La población de estudio estuvo conformada por 5537 estudiantes de siete instituciones educativas públicas de la ciudad de Pasto, entre las edades de 10 a 17 años. Con la aplicación de una fórmula estadística donde, con un grado de confianza del $95 \%$, probabilidad de éxito del 0.5 y un margen de error del 0.05 , se obtuvo la muestra, que quedó conformada por un total de 774 estudiantes, a partir de la afijación proporcional que permitió que a cada institución educativa le correspondiera una muestra proporcional, según la población que había presentado.

Una vez seleccionada la muestra, la aplicación del muestreo probabilístico aleatorio simple permitió que cada unidad tuviera la misma posibilidad de ser incluida; por lo tanto, la secuencia de acciones a seguir fue: tener en cuenta el marco muestral, que consistió en obtener una lista real de los estudiantes de acuerdo con la edad de 10 a 17 años; se determinó el número que integró la muestra y para ello se utilizó el azar por sorteo o rifa, anotando cada uno de los números individualmente y en secuencia, en pedazos de papel, hasta completar el número que compuso la muestra.

Posteriormente se tomaron las unidades correspondientes a la muestra, donde cada número indicaba la unidad que formó parte de la misma, teniendo en cuenta criterios de inclusión como que los jóvenes pertenecieran a las siete instituciones educativas escogidas y que estuvieran en los rangos de edad que corresponden a la etapa de la adolescencia. Criterios de exclusión, como que el estudiante hubiese sido trasladado a otro colegio en el momento de realizar la encuesta, no estuvieron presentes por diferentes causas.

La información se obtuvo mediante la aplicación de un cuestionario adaptado y elaborado a partir del instrumento Bullying Intimidación y Maltrato entre el alumnado de Avilés José María 2002 (9). Este instrumento es un formato impreso destinado a obtener respuestas sobre el problema de investigación, donde el consultado diligenció 21 preguntas: las primeras 10 correspondieron a selección múltiple tipo única respuesta, relacionadas con la dimensión demográfica y social; a partir de la pregunta 11, las dimensiones relacionadas con causas, tipo de agresión y conducta seguida ante un problema o conflicto, se codificaron a través de ítems tipo Likert, con respuestas de nunca, algunas veces, casi siempre y siempre. 
La validez y confiabilidad del instrumento se determinó con la validez de contenido, teniendo en cuenta el juicio que dieron expertos en el tema, quienes valoraron la matriz de consistencia a partir del problema, objetivos y variables y determinaron la coherencia entre ellos. Además, se realizó una prueba piloto que se aplicó a adolescentes de instituciones educativas con características similares a la muestra de estudio, con un porcentaje de aplicación del 10\% de la muestra.

El instrumento fue autodiligenciado en un contexto grupal, con la participación de las investigadoras y coinvestigadores, además de la presencia del coordinador académico o docente tutor de grupo. Se entregó el cuestionario para que los participantes procedieran a dar respuesta a las preguntas planteadas en el mismo -previamente se les había dado a conocer el propósito del estudio y se resolvieron las dudas-.

Se analizó la información utilizando el programa SPSS versión 20, paquete estadístico que permite trabajar con bases de datos de gran tamaño, analizarlas con estadísticas y representarlas con gráficas y tablas, además de ordenar y recodificar las variables.

Con respecto a los aspectos éticos, se solicitó autorización a cada una de las instituciones públicas de educación media, teniendo en cuenta que las investigadoras dieron a conocer a los rectores el propósito de la investigación. Posteriormente, para la recolección de la información, se solicitó el consentimiento informado a los padres de familia y el asentimiento informado a los menores de edad.

Igualmente, se tuvo en cuenta lo establecido en la Resolución No. 008430 de 1993 (11) en la cual se establecen las normas científicas, técnicas y administrativas para la investigación en salud. La investigación se realizó bajo los principios éticos expresados en la Ley 911 del 2004 (11) y la Ley 266 de 1996 (12).

\section{OBJETIVOS}

\section{Objetivo general}

Determinar el tipo de agresión que resulta de un problema o conflicto en adolescentes de siete instituciones públicas de educación media de San Juan de Pasto, Nariño, periodo 2013-2014.

\section{Objetivos específicos}

- Identificar el tipo de agresión física y/o verbal que se da entre los adolescentes.

- Identificar los lugares donde se presenta la agresión entre los adolescentes.

- Identificar las causas que llevaron a los adolescentes a realizar agresiones.

\section{RESULTADOS Y DISCUSIÓN}

Los adolescentes participantes en el estudio respondieron una serie de preguntas que llevaron a interpretar el tipo de agresión que se presenta en siete colegios de la ciudad de San Juan de Pasto.

\section{Tipo de agresión}

Entre las agresiones físicas relevantes, la Tabla 1 muestra que los adolescentes encuestados nunca han recibido golpes con el $66.7 \%$, mientras que el $25.3 \%$ los ha recibido algunas veces y casi siempre. Lo mismo sucede con recibir patadas y participar en peleas de grupos, donde los porcentajes se relacionan con un $62.5 \%$ y $68.5 \%$ respectivamente. Un bajo porcentaje de adolescentes participa en menor proporción o no participa en situaciones de agresión.

Tabla 1. Agresiones físicas entre adolescentes.

\begin{tabular}{|c|c|c|c|c|c|c|c|c|}
\hline $\begin{array}{c}\text { Ha } \\
\text { reci- } \\
\text { bido } \\
\text { golpes }\end{array}$ & FA & FR & $\begin{array}{c}\text { Ha } \\
\text { reci- } \\
\text { bido } \\
\text { pata- } \\
\text { das }\end{array}$ & FA & FR & $\begin{array}{c}\text { Ha } \\
\text { partici- } \\
\text { pado de } \\
\text { peleas } \\
\text { entre } \\
\text { grupos }\end{array}$ & FA & FR \\
\hline Nunca & 527 & 66,7 & $\begin{array}{l}\text { Nun- } \\
\text { ca }\end{array}$ & 486 & 62,5 & nunca & 533 & 68,5 \\
\hline $\begin{array}{l}\text { algu- } \\
\text { nas } \\
\text { veces }\end{array}$ & 158 & 20,3 & $\begin{array}{l}\text { algu- } \\
\text { nas } \\
\text { veces }\end{array}$ & 189 & 24,3 & $\begin{array}{l}\text { algunas } \\
\text { veces }\end{array}$ & 132 & 17 \\
\hline $\begin{array}{l}\text { casi } \\
\text { siem- } \\
\text { pre }\end{array}$ & 37 & 5.0 & $\begin{array}{l}\text { casi } \\
\text { siem- } \\
\text { pre }\end{array}$ & 52 & 6,7 & $\begin{array}{l}\text { casi } \\
\text { siempre }\end{array}$ & 41 & 5,3 \\
\hline $\begin{array}{l}\text { Siem- } \\
\text { pre }\end{array}$ & 25 & 3.2 & $\begin{array}{l}\text { Siem- } \\
\text { pre }\end{array}$ & 22 & 2.8 & siempre & 41 & 4 \\
\hline $\begin{array}{l}\text { No } \\
\text { res- } \\
\text { ponde }\end{array}$ & 27 & 4.8 & $\begin{array}{l}\text { No } \\
\text { res- } \\
\text { ponde }\end{array}$ & 25 & 3.7 & $\begin{array}{l}\text { No res- } \\
\text { ponde }\end{array}$ & 27 & 5.2 \\
\hline Total & 774 & 100 & Total & 774 & 100 & Total & 774 & 100 \\
\hline
\end{tabular}

Fuente: Instrumento Tipo de Agresión, 2013. 
En contraste con lo evidenciado anteriormente, la Tabla 2 muestra que las agresiones verbales son el tipo de agresión más frecuente entre los adolescentes, donde colocar sobrenombres o apodos es realizado frecuentemente por el 61.3\%. Acerca de los adolescentes que dicen palabras ofensivas, el 55\% lo realiza siempre y casi siempre; hablar mal de otras personas es una conducta adoptada por el $73.4 \%$ de los estudiantes para agredir verbalmente a sus compañeros.
Es importante determinar los lugares más relevantes donde se presentan las situaciones de agresión, sean estas físicas o verbales, tal como lo indica la tabla 3 , en donde se evidencia que la utilización del salón de clase cuando no está el profesor lo referencia el $72.3 \%$ de los adolescentes; de igual forma, el 73.6\% utiliza los pasillos y baños del colegio para realizar estos actos; otro porcentaje representativo del $70.8 \%$ utiliza los patios del colegio, pero el mayor porcentaje con el $97.2 \%$ indica que las peleas se generan cerca del colegio, al salir de clase.

Tabla 2. Agresiones verbales entre adolescentes.

\begin{tabular}{|l|r|r|l|r|r|}
\hline $\begin{array}{c}\text { Te han dicho } \\
\text { palabras ofensivas }\end{array}$ & \multicolumn{1}{|c|}{ FA } & \multicolumn{1}{|c|}{ FR } & \multicolumn{1}{c|}{$\begin{array}{c}\text { Han } \\
\text { hablado mal } \\
\text { de ti }\end{array}$} & \multicolumn{1}{c|}{ FA } & \multicolumn{1}{c|}{ FR } \\
\hline nunca & 243 & 31,2 & nunca & 185 & 23,8 \\
\hline algunas veces & 326 & 41,9 & $\begin{array}{l}\text { algunas } \\
\text { veces }\end{array}$ & 340 & 43,7 \\
\hline casi siempre & 104 & 13,4 & casi siempre & 108 & 13,9 \\
\hline siempre & 81 & 10,4 & siempre & 123 & 15,8 \\
\hline Perdidos no responde & 20 & 3,1 & $\begin{array}{l}\text { Perdidos no } \\
\text { responde }\end{array}$ & 18 & 2,8 \\
\hline Total & 774 & 100 & Total & 774 & 100 \\
\hline
\end{tabular}

Fuente: Instrumento Tipo de Agresión, 2013.

Tabla 3. Lugares donde se presentan situaciones de agresión entre los adolescentes.

\begin{tabular}{|l|l|r|l|r|r|l|l|l|l|l|l|}
\hline $\begin{array}{l}\text { Porque } \\
\text { me pro- } \\
\text { vocaron }\end{array}$ & FA & FR & $\begin{array}{l}\text { Porque } \\
\text { otros/ } \\
\text { as me lo } \\
\text { hacen }\end{array}$ & FA & FR & Por molestar & FA & FR & $\begin{array}{l}\text { Por gastar } \\
\text { una broma }\end{array}$ & FA & FR \\
\hline Nunca & 428 & 55 & nunca & 529 & 68 & nunca & 445 & 57,2 & nunca & 478 & 61,4 \\
\hline $\begin{array}{l}\text { algunas } \\
\text { veces }\end{array}$ & 188 & 24,2 & $\begin{array}{l}\text { algunas } \\
\text { veces }\end{array}$ & 129 & 16,6 & algunas veces & 194 & 24,9 & $\begin{array}{l}\text { algunas } \\
\text { veces }\end{array}$ & 158 & 20,3 \\
\hline $\begin{array}{l}\text { casi } \\
\text { siempre }\end{array}$ & 47 & 6 & $\begin{array}{l}\text { casi } \\
\text { siempre }\end{array}$ & 36 & 4,6 & casi siempre & 46 & 5,9 & casi siempre & 43 & 5,5 \\
\hline Siempre & 74 & 9.5 & siempre & 33 & 4.2 & siempre & 44 & 5.7 & siempre & 50 & 6.5 \\
\hline $\begin{array}{l}\text { No res- } \\
\text { ponde }\end{array}$ & 37 & 5.3 & $\begin{array}{l}\text { No res- } \\
\text { ponde }\end{array}$ & 47 & 6.6 & No responde & 45 & 6.3 & No responde & 45 & 6.3 \\
\hline Total & 774 & 100 & Total & 774 & 100 & Total & 774 & 100 & Total & 774 & 100 \\
\hline
\end{tabular}

Fuente: Instrumento Tipo de Agresión, 2013.

La tabla 4 aborda las causas que llevan a situaciones de agresión entre los adolescentes, donde el 55\% refiere que la provocación no es el factor que lleva a las peleas frente a un $40 \%$ que siente que esta sí es la causa común. Un $32 \%$ piensa que la causa generadora de violencia es porque otras personas lo está agrediendo, mientras que quienes lo hacen por molestar y gastar una broma equivalen al $57.2 \%$ y el $61.4 \%$, respectivamente. 
Tabla 4. Participación de los adolescentes en situaciones de agresión hacia compañeros /as ¿por qué lo hizo?

\begin{tabular}{|c|c|c|c|c|c|c|c|c|c|c|c|}
\hline $\begin{array}{c}\text { En la } \\
\text { clase } \\
\text { cuando } \\
\text { no está el } \\
\text { profesor/a }\end{array}$ & FA & FR & $\begin{array}{c}\text { En los } \\
\text { pasillos } \\
\text { y baños } \\
\text { del } \\
\text { colegio }\end{array}$ & FA & FR & $\begin{array}{c}\text { En los } \\
\text { patios }\end{array}$ & FA & FR & $\begin{array}{c}\text { Cerca } \\
\text { del } \\
\text { colegio, } \\
\text { al salir } \\
\text { de clase }\end{array}$ & FA & FR \\
\hline nunca & 188 & 24,2 & nunca & 178 & 22,9 & nunca & 192 & 24,7 & nunca & 100 & 12,9 \\
\hline $\begin{array}{c}\text { algunas } \\
\text { veces }\end{array}$ & 349 & 44,9 & $\begin{array}{c}\text { algunas } \\
\text { veces }\end{array}$ & 323 & 41,5 & $\begin{array}{c}\text { algunas } \\
\text { veces }\end{array}$ & 288 & 37 & $\begin{array}{c}\text { algunas } \\
\text { veces }\end{array}$ & 197 & 25,3 \\
\hline $\begin{array}{c}\text { casi } \\
\text { siempre }\end{array}$ & 113 & 14,5 & $\begin{array}{c}\text { casi } \\
\text { siempre }\end{array}$ & 147 & 18,9 & $\begin{array}{c}\text { casi } \\
\text { siempre }\end{array}$ & 169 & 21,7 & $\begin{array}{c}\text { casi } \\
\text { siempre }\end{array}$ & 175 & 22,5 \\
\hline siempre & 100 & 12.9 & siempre & 103 & 13.2 & siempre & 94 & 12.1 & siempre & 288 & 37 \\
\hline $\begin{array}{c}\text { No } \\
\text { responde }\end{array}$ & 24 & 3.5 & $\begin{array}{c}\text { No } \\
\text { responde }\end{array}$ & 23 & 3.9 & $\begin{array}{c}\text { No } \\
\text { responde }\end{array}$ & 31 & 4.5 & $\begin{array}{c}\text { No } \\
\text { responde }\end{array}$ & 14 & 2.3 \\
\hline Total & 774 & 100 & Total & 774 & 100 & Total & 774 & 100 & Total & 774 & 100 \\
\hline
\end{tabular}

Fuente: Instrumento Tipo de Agresión, 2013.

El bullying es un fenómeno que está presente de manera reiterada en cualquier institución educativa y que causa problemas de salud en los individuos afectados (13). Los adolescentes participantes en este estudio sufren problemáticas de agresión y violencia, fenómeno que les puede traer consecuencias académicas, sociales, personales, individuales y sicológicas.

Es importante comprender la dinámica del maltrato entre iguales, definiendo los términos agresión y violencia. La agresión es una conducta interpersonal cuya intención es herir o causar daño simbólico, verbal o físico a una persona que no desea sufrir esa suerte y que, de hecho, provoca daño real. La violencia es la agresión que tiene como objetivo causar daño físico extremo, como la muerte o graves heridas (14).

Por ello, la violencia escolar dentro y fuera de las aulas de clase es un problema que afecta el desarrollo del adolescente que lo padece. Aquí resulta de vital importancia el papel desempeñado por la escuela y la familia al ofrecer las herramientas básicas necesarias para la vida adulta independiente, en una sociedad donde se poseen derechos, responsabilidades, deberes y logros para la realización social, personal, espiritual y profesional.

Según el tipo de agresión, los adolescentes encuestados, a pesar de que son muy pocos los que han recibido golpes, patadas o a participado en peleas de grupos, en una mínima proporción sí se han visto afectados por estas situaciones, constituyéndose en hechos que atentan contra la integridad de la persona y que son fuente principal de conflicto.

Benavides (15) afirma que "en el colegio, la violencia está de moda, ya que casi un $6 \%$ de los alumnos han vivido el fenómeno conocido como bullying, que convierte a algunos escolares en víctimas de sus propios compañeros".

En España, Serrano e Iborra (16) mencionan que el porcentaje de víctimas de maltrato físico ha aumentado entre el año 2000 y el 2005, tanto en lo que se refiere a las situaciones de violencia que se dan de manera ocasional, como las que se dan de manera habitual.

Machado y Guerra (17), al indagar acerca de la participación en peleas con otros estudiantes del plantel, exponen que el $46 \%$ respondió que sí lo han hecho dentro del plantel y el $53 \%$ dijo que no ha peleado. Se puede inferir, entonces, que los estudiantes que pelean en grupo representan una porción significativa, lo cual se atribuye a un modo de establecer pertenencia, identidades y lealtades grupales mediante peleas en grupo. 
Tipo de agresión resultado de un problema y conflicto en la población adolescente de siete instituciones públicas de educación media de San Juan de Pasto • Claudia Amanda Chaves, Rosa Magaly Morales-Chincha, Nohora del Carmen Ortega-Cadena

ISSN 1794-9831

E-ISSN 2322-7028

Vol. 12 No. 1

Ene - Jun 2015

Cúcuta, Colombia
Es importante señalar que los resultados son similares a los datos encontrados por El Tiempo (18), en la consulta realizada a 273 escolares, donde el $70 \%$ respondió que ha sido víctima de agresiones verbales por parte de compañeros del colegio, el $18 \%$ de agresiones físicas y un $14 \%$ ha recibido amenazas y presiones para comportarse como lo exigen sus compañeros.

De la misma forma Ghiso (19) menciona que es común encontrar en las instituciones educativas conductas como intimidaciones, agresiones físicas, verbales y psicológicas, las cuales son ejercidas por individuos o por grupos y siempre están dirigidas contra otros individuos o grupos que no pueden defenderse y que se ven limitados en la denuncia y en el ejercicio de sus derechos a la legítima defensa y compensación entre adolescnetes.

De acuerdo con los resultados de Smith y Tomas (20), la detención de mujeres estadounidenses por cargos de asalto y armas está aumentando rápidamente. Las mujeres violentas fueron, significativamente, más propensas a rechazar la escuela y percibir la disciplina escolar como injusta.

De igual manera, según los datos encontrados, se evidencia la presencia de agresiones verbales por parte de los compañeros en las siete instituciones educativas analizadas, en lo que se refiere a la práctica de conductas ofensivas como poner sobrenombres o apodos y decir palabras ofensivas; hablar mal se reconoce como el comienzo de una posible agresión física que puede suceder tras una agresión de tipo verbal, lo cual se refleja en porcentajes considerables dentro del estudio.

Lo anterior es similar al estudio realizado por Plata et al. (21), el cual menciona que, dentro de los testigos, se observa con mayor frecuencia el acoso de tipo verbal, en mayor proporción el de poner apodos y hablar mal del otro. Igualmente, los datos ponen de manifiesto que la situación del bullying pasa desapercibida para el adulto, ya que los observadores no reportan esta situación a los profesores o los padres, sino a sus amigos o amigas.

Así mismo, de acuerdo con los datos del Gobierno Español (22), el porcentaje de incidencia total de alumnos víctimas de insultos pasa del $39,1 \%$ al $27 \%$ y el de víctimas de frases ofensivas del $37,7 \%$ al $26 \%$.
Se determinó así que la agresión verbal, la agresión física indirecta y golpear integran los mayores porcentajes dentro del alumnado que se confesó como agresor, frente a quienes se declararon como víctimas.

En este sentido, las instituciones educativas deben buscar caminos que lleven a la convivencia sana y a la generación de escenarios favorables que contribuyan a la formación de los estudiantes y su desarrollo personal; no obstante, hay que tener en cuenta que dicho compromiso debe ser liderado por el director de la institución, en conjunto con los docentes y padres de familia.

Desde hace más de una década la convivencia y disciplina escolar viene siendo un fenómeno que preocupa tanto a los profesores como a los directivos de las instituciones. Esto surge por la frecuencia con la que suceden hechos que alteran y rompen la armonía y convivencia en las aulas y centros educativos, y por la dificultad de encontrar soluciones idóneas y eficaces para superar el problema (23).

El tema del maltrato -físico o verbal- que se presenta en las instituciones educativas como conducta agresiva tiene varias formas de expresión y es el adolescente quien, a través de una ataque convertido en lesión u ofensa, trata en muchas ocasiones de llamar la atención o protestar ante el mundo. Es aquí donde diversos sectores deben intervenir para convertir aquellas expresiones de agresión y violencia de los estudiantes en reacciones de amistad, respeto y mutua confianza que lleven a las partes a evitar situaciones de enfrentamientos dolorosos.

Para Quijano (24), deben existir "unas excelentes prácticas que promuevan el desarrollo de competencias en los estudiantes; donde se hace necesario trabajar conocimientos, habilidades y actitudes para formar ciudadanos comunicados, para poder desempeñar un papel activo tanto en su núcleo escolar como familiar".

Lo más importante de todo es hacer frente a la problemática del bullying, realizando seguimiento permanente a los adolescentes víctimas y victimarios, como también a los testigos, para que sean ellos quienes denuncien las agresiones y para que este tipo de problemas no pase a otro nivel de violencia. 
Teniendo en cuenta que los estudiantes manifiestan haber participado en algún evento violento (peleas), se debe analizar este hecho desde su influencia en el comportamiento del adolescente y su consecuente contribución en la generación de conflictos. Con ello se pueden establecer parámetros eficientes que busquen promover un entorno escolar deseable, acorde con la misión institucional y que promueva los objetivos propuestos por el plantel educativo.

La violencia verbal es más grave en la relación entre estudiantes. Al analizar la presencia de este tipo de maltrato, se puede evidenciar que en las instituciones educativas muchos alumnos son víctimas de apodos que les ponen sus compañeros con el fin de agredirlos. Es notorio que existe un alto porcentaje de estudiantes que tiende a usar apodos o sobrenombres para referirse a sus compañeros; por eso, es necesario analizar qué influencia tiene dicho aspecto, con el fin de evitar confrontaciones entre estudiantes de la institución.

Con relación a este tema, Castillo (25) afirma que "los acosadores utilizan distintas formas como los apodos, mofas, insultos y habladurías, que generan intimidación, exclusión $\mathrm{y}$, en ocasiones, serios problemas psicológicos y sociales que provocan dificultades en la convivencia y en la adaptación social".

Las agresiones verbales son comunes en la cultura y en la población objeto de estudio, donde se identificaron porcentajes representativos que abren la posibilidad de generación de conflictos y presencia de problemas que traigan consecuencias tanto a los adolescentes como a las instituciones educativas.

Para Jares (26), "el conflicto o el desacuerdo se interpreta como una desviación de la tarea, derivada de la reacción emocional, más que como la oposición de aquellos que definen la tarea del colegio de forma distinta. Por consiguiente, el conflicto es considerado como un elemento que acaba por perjudicar gravemente el normal funcionamiento de la organización".

Julien, de acuerdo con De Souza(27), define el conflicto como "un enfrentamiento o choque intencional entre dos individuos o grupos de la misma especie que manifiestan una intención hostil, generalmente acerca de un derecho, y que buscan romper la resistencia del próximo, usando la violencia, la cual podría llevar al aniquilamiento físico del otro".

En virtud de lo anterior, uno de los retos de la educación de hoy se direcciona hacia la preservación y sostenibilidad de una buena convivencia que influya en que los estudiantes de un plantel educativo se identifiquen con su institución y generen sentido de pertenencia, de la mano del aprendizaje como experiencia emocionalmente significativa, y que el trabajo colaborativo logre empoderar a los niños, jóvenes y adolescentes generándoles mayor autoestima, valoración y confianza en sí mismos y los demás.

Los resultados obtenidos evidencian que un gran porcentaje de adolescentes habla mal de otras personas, lo cual afecta las relaciones interpersonales de los estudiantes y puede ser uno de los factores que altere el ambiente escolar y, por consiguiente, genere conflictos en las aulas y el entorno.

Resultados semejantes obtuvieron los autores Arellano y Tinedo (28), quienes afirman que "el conflicto es inherente a las condiciones humanas $\mathrm{y}$, por ende, al comportamiento como elemento connatural e inseparable de las relaciones entre las personas y los grupos. En esta relación se manifiestan contradicciones, pugnas y enfrentamientos, pero igualmente acuerdos, afinidades y conciliaciones que potencian al hombre como ser capaz de articularse consigo mismo y con los otros".

Es fundamental y recomendable que un profesional realice acompañamiento y orientación permanente al estudiante con el fin de mejorar continuamente el clima escolar, el cual conduce a un ambiente de armonía y construcción de escenarios favorables para desarrollar unas buenas prácticas pedagógicas, a corto y mediano plazo.

Por otra parte, en las instituciones educativas, son muchos los causantes de problemas de comunicación: las características que posee cada individuo pueden desembocar en inadecuadas formas de relacionarse, la empatía o su ausencia, el estrés, las actitudes y los problemas personales generan conflictos, siendo fundamental que el docente trabaje en identificar aspectos o elementos que deterioran las relaciones, para impedir que se desarrolle el conflicto.

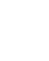


ISSN 1794-9831

E-ISSN 2322-7028

Vol. 12 No. 1

Ene - Jun 2015

Cúcuta, Colombia
Al respecto Garaigordobil y Oñedera (14) señalan que la violencia que ocurre como consecuencia del maltrato entre iguales es variada y ocurre en diversos tipos de conductas, es prologada en el tiempo -no esporádico- y sucede en espacios donde se convive con el agresor, tales como la escuela, y es producido por un individuo o grupo de individuos frente a quienes la víctima se siente indefensa.

Benítez (29) plantea "la existencia de las agresiones directas o explicitas frente a las encubiertas, siendo las ultimas -las de carácter verbal, como poner motes y expandir rumores-las más frecuentes". De igual forma De Zubiria (30) contempla que "uno de cada cuatro de los estudiantes latinoamericanos había sido agredido verbalmente por sus compañeros"

Datos similares se encuentran en el estudio de Collell y Escude (31), quienes afirman que la poca atención que se suele prestar al maltrato verbal y, especialmente, a la exclusión social que éste genera, son expresiones poco consideradas y pueden gozar de cierta permisividad por parte de los compañeros e incluso de los adultos, quienes suelen asimilar el maltrato con la violencia física. Contrariamente a lo que puede suponerse, las conductas de maltrato verbal y la exclusión social tienen, a largo plazo, un peor pronóstico para quien las sufre, por encima de la agresión abierta.

Con los resultados de este estudio, se realizó el análisis de las agresiones verbales a través de la presencia de agresiones en el plantel educativo por parte de los compañeros(as) de curso, relacionadas con la frecuencia en que sucede la agresión verbal. En consonancia con lo anterior, los resultados muestran que, en los siete colegios investigados, fue el maltrato verbal la expresión de violencia más frecuente que se presentó en el transcurso de tiempo y que allí se deriva la violencia física, traducida en patadas y golpes.

\section{Lugares donde se presenta la agresión}

Los datos revelan que la mayoría de los adolescentes consultados manifiestan que las situaciones de agresión se presentan en el salón de clase, cuando no está el profesor. Es ahí donde son violentados y muchos estudiantes aprovechan la situación para atacar y son objeto de abuso por los mismos compañeros. Solamente una menor proporción de ellos no ha sido víctima de esta situación.
El Fondo de Bienestar Social de la Contraloría General de la Republica (32) refiere que se presentan diversas manifestaciones de matoneo en los colegios, como la de exigir dinero a cambio de no golpear al compañero, el maltrato físico con golpes, patadas y empujones, la burla, las amenazas y el poner apodos crueles e insultantes, fenómeno que siempre ocurre cuando no está el profesor en el aula de clase.

Se pone en evidencia el hecho que los adolescentes requieren una vigilancia continua y apoyo por parte de diferentes sectores para trabajar con las víctimas $\mathrm{y}$ victimarios $\mathrm{y}$, con ello, intervenir de diferentes maneras para cambiar el modo de pensar y de actuar de los adolescentes; aquí el papel desempeñado por el docente es claro y vital para detectar el maltrato dentro de las instituciones, dado que los profesores se relacionan más con los estudiantes.

Cobo (33) encontró que cuando se produce el bullying, el agresor escoge cualquier sitio del colegio que esté apartado (baños, pasillos vacíos, vestuarios, comedores, transporte público y patios, entre otros) para practicarlo. Sin embargo, todo empieza en las aulas: por lo general actúa cuando el profesor se ausenta, aunque, en los casos más graves, incluso puede actuar delante del docente.

Se encontró que casi la totalidad de los adolescentes de las siete instituciones objeto de estudio afirmó que los pasillos, baños y alrededores del colegio, al salir de clase, son los sitios donde el agresor aprovecha, debido a que no está vigilado, para violentar a sus compañeros y crear un problema o conflicto.

Estos hallazgos son similares a los presentados por Brizzuela (34), quien afirma que "las aulas, los pasillos, el baño y el patio o la salida del colegio son el escenario donde los bullies ejecutan su propia ley, sin que los profesores puedan evitarlo".

Existe una grave problemática cuando los adolescentes encuentran fuera del colegio, al salir de clase, el ambiente propicio para generar algún tipo de agresión, haciendo que se pierda todo tipo de valores, porque al agredir a otros se produce desestabilidad emocional y física en el agredido, ocasionando que permanentemente se sienta amenazado y en una ambiente hostil que, posteriormente, lo puede llevar a adoptar conductas inadecuadas. 
Montero (35) refiere que los adolescentes encuentran, al salir de clase, un modo de reconocer y resolver situaciones y problemas propios de esta etapa de la vida; sienten a los personajes como gente real que puede producir algún tipo de socialización en la construcción de sus valores, normas y actitudes.

Según García (36), "el fenómeno de la violencia transciende la conducta individual y se convierte en un proceso interpersonal, porque afecta al menos a tres protagonistas: quien la ejerce, quien la padece y quien la contempla sin poder o querer evitarla". Los jóvenes son copia de lo que ven; y al vivir cotidianamente esta situación, aprenden de ella y la ejecutan.

Otro sitio donde los adolescentes encuentran el ambiente apto para la agresión es el patio de las instituciones educativas: la mayor parte de los estudiantes manifestó aprovechar este lugar para atacar a los compañeros y así someterlos a maltrato físico, verbal y psicológico.

Lo anterior se confirma con el trabajo de Cano y Luaces (37), quienes mencionan en su estudio que "el lugar más frecuente donde se produce la agresión, en la primaria y la secundaria, es el patio". Allí el agresor es impulsivo y puede ser físicamente más fuerte, ejerciendo con ello el maltrato sobre sus pares.

Resultados semejantes se presentan en el estudio de Aznar et al. (38) donde se afirma que "el panorama de conflictividad que se presenta en los centros escolares se refleja a nivel de los distintos espacios escolares, como las aulas, pasillos, aseos, vestuarios, patio, comedor, etc.".

Al comparar los resultados, se demuestra que en las siete instituciones de educación media de Pasto el panorama es igual que en otros colegios de otras ciudades, lo cual es acorde con el planteamiento teórico que considera que el patio es uno de los lugares más apropiados para que se presente una situación de agresión, pero que cuando dicha agresión se torna más violenta se recurre a otros sitios.

Aznar et al. (38) afirman que "cuando la situación avanza, el agresor no actúa sólo en el colegio, sino que espera a la víctima en diferentes sitios cerca del colegio o incluso pacta horas para verse y actuar".

\section{Causas de las agresiones}

Entre las motivaciones para la participación en situaciones de agresión hacia compañeros/as se encuentra: porque lo provocaron, porque me lo hacen otros, por molestar, por gastar una broma, situaciones que están presentes dentro de la cotidianeidad del contexto educativo y ajenas al reconocimiento por parte de los docentes.

Es así como se evidencia que los estudiantes, algunas veces, generan este tipo de agresiones y esto, a su vez, deteriora las relaciones interpersonales y el ambiente académico, generando problemas de convivencia en las aulas escolares.

De hecho, todos los comportamientos, que en sí mismos no constituyen formas graves de violencia, por acumulación pueden llegar a hacer imposibles las actividades académicas y generar problemas mucho más importantes entre los mismos alumnos y entre los maestros y su alumnado. Para Salame (39), la falta de normas propicia que se confunda o se desplace la frontera entre lo que está bien y lo que está mal, y esto contribuye a la extensión de nuevas formas de violencia.

En este punto es fundamental que todas las instituciones educativas fortalezcan una formación basada en valores, puesto que las conductas agresivas interfieren en el buen funcionamiento de las prácticas pedagógicas $y$, de hecho, proyectan a la sociedad una imagen negativa del colegio.

Los resultados reflejan que la mayoría de los adolescentes no ha participado en alguna situación de agresión, ya que ellos no han sido provocados en el plantel educativo. Sin embargo, sí se han presentado estas situaciones y, por ello, deben tomarse medidas preventivas y ayuda profesional a fin de fomentar la tolerancia en el entorno escolar y disminuir este indicador para preservar el ambiente educativo.

Las agresiones causadas simplemente por molestar rodean en mínima proporción a la comunidad escolar, puesto que la mayoría nunca agrede a otros por molestar. Esto demuestra que los adolescentes pueden ser personas tolerantes y que, con sus actuaciones, demuestran el respeto hacia sus compañeros. Sin embargo, en un pequeño porcentaje, el acto de molestar sí se presenta, ante lo cual debe prestarse atención porque puede llevar a conductas graves. 
Tipo de agresión resultado de un problema y conflicto en la población adolescente de siete instituciones públicas de educación media de San Juan de Pasto • Claudia Amanda Chaves, Rosa Magaly Morales-Chincha, Nohora del Carmen Ortega-Cadena

ISSN 1794-9831

E-ISSN 2322-7028

Vol. 12 No. 1

Ene - Jun 2015

Cúcuta, Colombia
Se analizó como este tipo de comportamientos -la acción de realizar una broma- puede afectar el entorno escolar y la ejecución de unas óptimas prácticas pedagógicas, pues no es solamente un problema que afecta las relaciones interpersonales de los estudiantes sino que es un factor que, a corto plazo, puede incidir en el desarrollo de una sana convivencia. De esta forma, se evidenció que las agresiones no se dan sencillamente por gastar una broma, lo cual se ve reflejado en los bajos porcentajes de respuesta.

Vargas et al. (40) plantean al respecto, para resolver la problemática de conflictos, 5 estilos de resolución de conflictos: confrontación, compromiso, facilitación, presión y retirada.

Siguiendo el análisis del tema del maltrato entre iguales, el adolescente, dentro de la dinámica de la violencia, generalmente encuentra consecuencias para el agredido, para el agresor y el testigo, tales como bajo rendimiento académico y fracaso escolar, rechazo al colegio, relaciones sociales negativas, miedo, sumisión e insolidaridad, entre otros.

Frente a esta situación, es importante determinar las formas más comunes de solucionar las agresiones y conflictos entre compañeros, puesto que el adolescente debe fomentar habilidades sociales que le permitan desenvolverse en ambientes diversos, establecer relaciones interpersonales y de autocontrol, lo cual le garantiza salud mental en la adultez.

Estas competencias se aprenden y se practican en diversos ámbitos -redes sociales formadas por la familia, la escuela y la comunidad- que pueden o no funcionar como apoyo. Cuando funcionan adecuadamente, los niños y jóvenes se adaptan bien, tienen buen rendimiento y no son víctimas fáciles de eventos violentos; pero cuando no funcionan adecuadamente, se genera una ineficiente estructura que puede colapsar por su fragilidad. Díaz (41) afirma que el niño y el joven están cometiendo actos de agresión en ámbitos donde los adultos muchas veces han perdido su autoridad sobre ellos.

Es importante señalar que el tipo de violencia verbal o física existe y está latente todos los días en las diferentes instituciones educativas, acentuando el problema en unas instituciones más que en otras. Los adolescentes son quienes protagonizan y generan esta violencia. Las personas agredidas muchas veces no denuncian este problema por temor a seguir siendo maltratadas y esta falta de denuncia puede traer consecuencias lamentables.

Es fundamental que todos los sectores, tanto el educativo como el de salud, además de la familia y el gobierno, unan esfuerzos para poder ponerle freno a este tipo de violencia, mediante estrategias para prevenirla o tratar de que no genere consecuencias fatales. Lo ideal es que se trabaje en un modelo de atención integral donde cada uno de los sectores aporte para la prevención y mitigación de este problema, enseñando desde la infancia a evitar problemas y ayudando a resolverlos de la mejor manera, con tolerancia y mecanismos de resolución de conflictos.

Por la dinámica del maltrato entre iguales, el programa de enfermería hace frente a las nuevas tendencias del cuidado -entendido desde el desarrollo de la personay desde nuevas perspectivas propuestas por la salud pública, frente a los cambios políticos, sociales y económicos que promueven la participación en la toma de decisiones para favorecer la salud mental de las poblaciones.

Para la resolución de conflictos entre adolescentes se requiere el trabajo interdisciplinario, puesto que se debe intervenir este tipo de problemáticas no sólo desde acciones individuales sino desde la participación interdisciplinaria y de apoyo a grupos específicos. De ahí que se recomienda fomentar el trabajo desde la relación con el sector externo que involucre también a instituciones educativas, programas académicos de educación superior y padres de familia, sin olvidar la participación activa del adolescente en la formulación de nuevas propuestas encaminadas a contrarrestar el maltrato entre iguales.

Es así como la intervención de enfermería se caracteriza por brindar continuidad y seguimiento del cuidado, siendo fundamental fortalecer la consulta, propuesta formulada a las instituciones prestadoras de salud y encaminada a fomentar una consulta de enfermería desde el cuidado del niño y adolescente, con enfoque del cuidado desde la persona, desde la interrelación de la planificación del cuidado, cuyo principal objetivo estaría encaminado a fomentar la asistencia familiar, identificación de casos de maltrato entre iguales y seguimiento de los mismos.

Adicionalmente, es importante incluir en el plan de formación del profesional de enfermería un curso 
electivo que brinde conocimientos en resolución de conflictos, lo cual le permitirá adquirir herramientas de intervención específica en el momento que la o el enfermero identifique a un niño agresor o un niño agredido.

\section{CONCLUSIONES}

Con respecto al tipo de agresión que resulta de un problema o conflicto, se concluye que menos de la mitad de los estudiantes ha recibido golpes, patadas o ha participado en peleas de grupos; estas acciones son atribuidos a las malas relaciones interpersonales. Por el contrario, una gran proporción de adolescentes menciona que le han puesto sobrenombres, le han dicho palabras ofensivas y han hablado mal de ellos, es decir, agresiones de tipo verbal.

La presencia de agresiones verbales por parte de los compañeros, en las siete instituciones educativas participantes en la investigación, se reconoce como el comienzo de una posible agresión física que puede suceder tras esa agresión de tipo verbal, siendo ésta última la más reconocida por los adolescentes y la más frecuente en las instituciones.

El tipo de agresión, sea física o verbal, incide negativamente en el entorno escolar y desencadena una serie de situaciones que no son favorables para ejercer unas excelentes prácticas pedagógicas, pues afecta las relaciones interpersonales entre los estudiantes, el desempeño de las actividades académicas y se perturba de manera individual o colectiva a un grupo de personas.

Las agresiones físicas como pegar patadas, empujar o dar golpes, fueron reconocidas en menor frecuencia por los adolescentes de las sietes instituciones educativas. Sin embargo, persiste el interrogante de si los encuestados dijeron toda la verdad con respecto a las preguntas realizadas o prefirieron seguir encubriendo, de una u otra manera, este tipo de agresiones, las cuales se acrecientan cada vez más dentro y fuera de las instituciones educativas.

El lugar más frecuentes para llevar a cabo el matoneo entre compañeros es el salón de clase, cuando no está el profesor. También se da en los baños, en los patios, en el recreo cuando hay muchos estudiantes disfrutando de este espacio y los docentes compartiendo un momento de receso, además de fuera del colegio, al salir de este.
Las causas más sobresalientes de las agresiones entre los adolescentes están vinculadas a provocaciones o porque otros compañeros agreden primero, por gastar una broma y otros lo hacen por molestar. Estas conductas han llevado al joven a actuar de forma violenta, fenómeno que trae como consecuencia la provocación del adolescente, situación en donde los docentes y los compañeros de clase no son ajenos.

La intimidación de los compañeros durante la infancia y la adolescencia afecta a una minoría significativa de los individuos; aunque es una experiencia adversa, no parece ser una variedad psicológica de corto potencial y largo plazo, así como de secuelas somáticas.

La violencia entre adolescentes dentro y fuera del aula escolar es un problema que afecta el desarrollo de los jóvenes. Por ello, los contextos como la escuela y la familia deben ofrecer las herramientas necesarias para la vida adulta independiente, en una sociedad donde se posee derechos, responsabilidades, deberes y logros para la realización social, personal, espiritual y profesional.
ISSN 1794-9831

E-ISSN 2322-7028 Vol. 12 No. 1

Ene - Jun 2015

Cúcuta, Colombia 
Tipo de agresión resultado de un problema y conflicto en la población adolescente de siete instituciones públicas de educación media de San Juan de Pasto • Claudia Amanda Chaves, Rosa Magaly Morales-Chincha, Nohora del Carmen Ortega-Cadena

ISSN 1794-9831

E-ISSN 2322-7028

Vol. 12 No. 1

Ene - Jun 2015

Cúcuta, Colombia

\section{REFERENCIAS BIBLIOGRÁFICAS}

1. Borrás T. Adolescencia: definición, vulnerabilidad y oportunidad. Correo Científico Medico. [Internet]. 2014 [consultado 23 de marzo de 2015]; 18(1):5-7. Disponible en: http://scielo.sld.cu/pdf/ccm/v18n1/ccm02114.pdf

2. Gómez A, Gala FJ, Lupiani M, Bernalte A, Miret MT, Lupiani S, et al. El "bullying" y otras formas de violencia adolescente. Cuadernos de Medicina Forense. [Internet]. 2007 [consultado 14 de junio de 2012]; 13(49-49):165177. Disponible en: http://scielo.isciii.es/pdf/cmf/n48-49/art05.pdf

3. Ramos L. La agresividad de los adolescentes de Educación Secundaria. [Tesis en Internet]. Chihuahua: Centro Chihuahuense de Estudios de Posgrado; 2010 [consultado 11 de enero de 2011]. Disponible en: http: http://www. cchep.edu.mx/docspdf/cc/119.pdf

4. Paredes M, Lega L, Cabezas H, Ortega M, Medina Y, Vega C. Diferencias transculturales en la manifestación del Bullying en Estudiantes de Escuela Secundaria. Revista Latinoamericana de Ciencias Sociales, Niñez y Juventud. [Internet]. 2011 [consultado 9 de septiembre de 2012]; 2(9):768-768. Disponible en: http://www. scielo.org.co/pdf/rlcs/v9n2/v9n2a18.pdf

5. SalmivallI C, Kärnä A, Poskiparta E. Counteracting bullying in Finland: The KiVa program and its effects on different forms of being bullied. International Journal of Behavioral Development. [Internet] 2011 [consultado 30 de enero de 2011]; 35(5): 405-411. Disponible en: http: http://jbd.sagepub.com/content/ear ly/2011/07/19/0165025411407457

6. De Zubiria J, Castilla D, Peralta D. La violencia escolar entre compañeros en una muestra de colegios privados de Bogotá. [Internet]. Bogotá: Publicaciones y artículos; 2009. [Consultado 26 de febrero de 2011]. Disponible en: http://www.institutomerani.edu.co/publicaciones/articulos/2009/articulo_ divulgativo_violencia_escolar_ peralta_castilla.pdf

7. Redondo J, Guevara E. Diferencias de género en la prevalencia de la conducta prosocial y agresiva en adolescentes de dos colegios de la ciudad de Pasto - Colombia. Revista Virtual Universidad Católica del Norte [Internet]. 2012 [Consultado 19 de febrero de 2013]; (36):173-192. Disponible en: http://revistavirtual.ucn.edu.co/index. $\mathrm{php} /$ RevistaUCN/article/view/376

8. República de Colombia. Ministerio de Educación. Ley 1620 de 2013, marzo 25, por la cual se crea el Sistema Nacional de Convivencia Escolar y Formación para el Ejercicio de los Derechos Humanos, la Educación para la Sexualidad y la Prevención y Mitigación de la Violencia Escolar. [Internet]. Bogotá: Mineducacion; 2013. [consultado 25 de marzo de 2015]. Disponible en: http://www.mineducacion.gov.co/1621/w3-article-322486. $\mathrm{html}$

9. Aviles J. Bullying intimidación y maltrato entre el alumnado. Educación en valores [Internet]. 2013 [Consultado 12 de marzo de 2011]. Disponible en: http://www.educacionenvalores.org/spip.php?article1032.

10. República de Colombia. Ministerio de Salud. Resolución 008430 de 1993, octubre 4, por la cual se establecen las normas científicas, técnicas y administrativas para la investigación en salud. San Fe de Bogotá: El Ministerio; 1993.

11. República de Colombia. Ministerio de Educación. Ley 911 de 2004, octubre 5, por la cual se dictan las disposiciones en materia de responsabilidad deontológica para el ejercicio de la profesión de Enfermería en Colombia. [Internet]. Bogotá: El Ministerio; 2004 [consultado 15 de octubre de 2012]. Disponible en: http:// www.mineducacion. gov.co/1621/articles-105034_archivo_pdf

12. República de Colombia. Gobierno Nacional. Ley 266 de 1996, enero 25, por cual la se reglamenta la profesión de Enfermería en Colombia y se dictan otras disposiciones. Diario oficial No. 42.710. [Internet]. 1996 [consultado 15 de octubre de 2012]. Disponible en: http://www.mineducacion.gov.co/1621/articles-105002_archivo_pdf.pdf

13. Sansone R, Sansone L. Bully victims: 2008 psychological and somatic aftermaths. Psychiatry (Edgmont) [Internet]. 2008 [consultado 15 de octubre de 2012]; 5(6):62-64. Disponible en: www.ncbi.nim.nih.gov/pmc/ar

14. Garaigordobil M, Oñedera JA. La Violencia entre iguales. Revisión teórica y estrategias de Intervención. Madrid: Pirámide Ed.; 2010.

15. Benavides I. Adolescencia y Violencia: ¿crisis o patología? Clínica de Psicología [Internet]. 2006 [consultado 15 de junio de 2013]. Disponible en: http://www.acosomoral.org/pdf/adolescencia_y_violencia.PDF

16. Serrano A, Iborra I. Violencia entre compañeros en la escuela. informe España. [Internet]. [consultado 15 de junio de 2013]. Disponible en: http://213.0.8.18/portal/Educantabria/RECURSOS/ Materiales/Biblestinv/ Informe_Violencia_entre_compa\% $\mathrm{C} 3 \% \mathrm{~B} 1 \mathrm{eros}$ _en_la_escuela.pdf

17. Machado J, Guerra J. Violencia en las escuelas. Centro Gumilla [Interrnet]. Argentina: Ministerio de educación; 2009 [consultado 30 de mayo de 2013]. Disponible en: https://sicsemanal.files.wordpress.com/2010/03/gumillainforme-violencia-en-las-escuelas.pdf 
18. El Tiempo. Alumnos, víctimas de agresión verbal en Cali. [Internet]. 9 de noviembre de 2012; Opinión: 5C. Disponible en: http://www.eltiempo.com/archivo/documento/CMS-12370706

19. Ghiso A. Naturalización de la intimidación entre escolares: un modo de construir lo social. Revistaumanizales. cinde.org.co [Internet]. 2010 [consultado 12 de mayo de 2013]. Disponible en: http://www.academia. edu/1297182/Naturalizacion_de_la_intimidacion_entre_escolares_un_modo_de_construir_lo_social

20. Smith $\mathrm{H}$, Thomas $\mathrm{S}$. Violent and non-violent girls: contrasting perceptions of anger experiences, school, and relationships. Healthcare. [Internet]. 2000 [consultado 15 de enero de 2011]; 21(5); 47-75. Disponible en: http:// informahealthcare.com/doi/pdf/10.1080/01612840050044285

21. Plata $\mathrm{C}$, Riveros M, Moreno J. Autoestima y empatía en adolescentes observadores, agresores y víctimas del bullying en un colegio del municipio de Chía. Psychologia. Avances de la disciplina. [Internet]. 2010 [consultado 24 de marzo de 2013]; 4(2): 99-112. Disponible en: http://www.scielo.org.co/scielo.php?script=sci_arttext\&pid $=$ S1900-23862010000200009

22. Defensor del Pueblo. Violencia Escolar: el maltrato entre iguales en la Educación Secundaria Obligatoria 19992006. [Internet]. Madrid: Defensor del Pueblo; 2007 [Consultado 19 de noviembre de 2012]. Disponible en: http://www.defensordelpueblo.es/es/Documentacion/Publicaciones/monografico/Documentacion/Informe_ violencia_escolar_ESO.pdf

23. García A. La disciplina escolar. Guía docente. [Internet]. España: Universidad de Murcia; 2008. [consultado 23 de noviembre de 2012]. Disponible en: https://www.google.com.co/search?q=La+disciplina+en+el+\%C3\%A1 mbito + escolar. $+\mathrm{Gu} \% \mathrm{C} 3 \% \mathrm{ADa}+$ docente\& $\mathrm{oq}=\mathrm{La}+$ disciplina $+\mathrm{en}+\mathrm{el}+\% \mathrm{C} 3 \% \mathrm{~A} 1 \mathrm{mbito}+$ escolar. $+\mathrm{Gu} \% \mathrm{C} 3 \% \mathrm{ADa}+$ docente\&aqs $=$ chrome..69i57.1982j0j7\&sourceid $=$ chrome\&es_sm $=0 \& i e=U T F-8$

24. Quijano A. Las Competencias comunicativas en la solución de conflictos en el aula. [Internet]. Pereira: Universidad Tecnológica de Pereira; 2007. [consultado 23 de octubre de 2012]. Disponible en: http://repositorio. utp.edu.co/dspace/bitstream/11059/983/1/30254C227c.pdf

25. Castillo L. El acosador escolar: de las causas, origen y manifestaciones a la pregunta por el sentido que le otorgan los actores. Magis. [Internet]. 2011 [consultado 12 de agosto de 2013]; 4(8): 1-14. Disponible en: http://revistas. javeriana.edu.co/index.php/MAGIS/article/view/3572

26. Jares J. El lugar del conflicto en la organización escolar. Revista iberoamericana de educación [Internet]. 1997 [consultado 22 de junio de 2013]; 4(15):3-56. Disponible en: http://www.rieoei.org/oeivirt/rie15a02.htm

27. De Souza L. Una mirada genérica de los conflicto. Contribuciones a las Ciencias Sociales CCCSS [Internet]. 2009 [consultado 24 de noviembre de 2014]. Disponible en: http://www.eumed.net/rev/cccss/04/lsb.htm

28. Arellano N, Tinedo Y. Asimetría Comunicativa: Fuente generadora de conflictos interpersonales entre docentes y estudiantes. Orbis. [Internet]. 2009 [consultado 3 de septiembre de 2013]; 7(19): 121-147. Disponible en: http:// www.redalyc.org/articulo.oa?id=70919155007

29. Benítez J, Justicia F. Maltrato entre iguales, descripción y análisis del fenómeno. Psycoeducativa [Internet]. 2006 [consultado 12 de septiembre de 2012]; 4(2):151-170. Disponible en: http://www.investigacion-psicopedagogica. org/revista/articulos/9/espannol/Art_9_114.pdf

30. De Zubiria-Samper J. La violencia en los colegios de Bogotá. [Internet]. 2009 [consultado 15 de enero de 2013]. Disponible en: http://www.institutomerani.edu.co/publicaciones/articulos/2009/La_violencia_en_los_colegios_ de_Bogot $\%$ C $3 \%$ A1.pdf

31. Collell J, Escude C. El acoso escolar: un enfoque psicopatológico. Anuario de Psicología Clínica y de la Salud. [Internet]. 2006 [consultado 4 de noviembre de 2014]; 2: 9-14. Disponible en: http://institucional.us.es/apcs/doc/ APCS_2_esp_9-14.pdf

32. Fondo de bienestar social de la Contraloría General de la República. ¿Sabes qué es el matoneo escolar o bullying? [Internet]. Bogotá: Contraloría General de la Nación; 2009. [consultado 15 de enero de 2013]. Disponible en: http://www.fbscgr.gov.co/?idcategoria $=2251$

33. Cobo S. Bullying - Jane Addams Midle School. [Internet]. 2013 [consultado 14 de julio de 2013]. Disponible en: http://www.bullying-jams.wikispaces.com/\%c2\%bfd\%c3\%b3nde+pasa $\% 3 \mathrm{f}$

34. Brizuela F. La Violencia en el ámbito escolar. Monografias.com [Internet]. 1999 [consultado 19 de febrero de 2013]. Disponible en: http://www.monografias.com/trabajos91/violencia-ambito-escolar/violencia-ambitoescolar.shtml

35. Montero Y. Estudio empírico sobre el serial juvenil «Al salir de clase»: sobre la transmisión de valores a los adolescentes. Revistacomunicar.com [Internet]. [consultado 22 de enero de 2013]. Disponible en: http://www. revistacomunicar.com/verpdf.php?numero $=25 \&$ articulo $=25-2005-070$

36. García F. Modelo Ecológico, Modelo Integral Conceptualización del desarrollo y de la atención temprana desde diferentes escuelas psicológicas. [Internet]. Murcia: Universidad de Murcia; 2001. [consultado 6 de mayo de 
Tipo de agresión resultado de un problema y conflicto en la población adolescente de siete instituciones públicas de educación media de San Juan de Pasto • Claudia Amanda Chaves, Rosa Magaly Morales-Chincha, Nohora del Carmen Ortega-Cadena

ISSN 1794-9831

E-ISSN 2322-7028 Vol. 12 No. 1 Ene - Jun 2015 Cúcuta, Colombia
2013]. Disponible en: http://www.sld.cu/galerias/pdf/sitios/rehabilitacion-temprana/modelo_ecologico_y_ modelo_integral_de_intervencion.pdf

37. Cano T, Luaces V. Bullying: Violencia en adolescentes y jóvenes. [Internet]. Montevideo: Divina Providencia; 2009 [consultado 24 de febrero de 2013]. Disponible en: http://ipes.anep.edu.uy/documentos/noticias_portada/ vinculo_abajo/cce/cano_b.pdf

38. Aznar I, Cáceres M, Hinojo F. Estudio de la violencia y conflictividad escolar en las aulas de clase de educación primaria a través de un cuestionario de clima clase: el caso de las provincias de Córdoba y Granada (España). Revista Iberoamericana sobre Calidad, Eficacia y Cambio en Educación - REICE. [Internet]. 2007 [consultado 11 de julio de 2013]; 5(1):164-177. Disponible en: http://www.redalyc.org/pdf/551/55100110.pdf

39. Salame C. Conflicto y violencia en el ámbito escolar. Revista de estudios sobre Juventud. [Internet]. 2003 [consultado 26 de mayo de 2013]; 7(19): 48-61. Disponible en: http://www.acosomoral.org/pdf/ ConflictoviolenciaenE1mbitoescolarCarlesSerra.pdf

40. Vargas A, Cabrera H, Rincón P. Estilos de solución de conflictos. Revista Latinoamericana de Psicología [Internet]. 2000 [consultado 16 de marzo de 2013]; 10(1): 21-25. Disponible en: http://www.redalyc.org/articulo. oa?id.

41. Díaz M. Convivencia escolar, disciplina y prevención de la violencia. [Internet]. Madrid: Universidad Complutense; 2012. [consultado 12 de mayo de 2013]. Disponible en: http://mariajosediaz-aguado.tk 